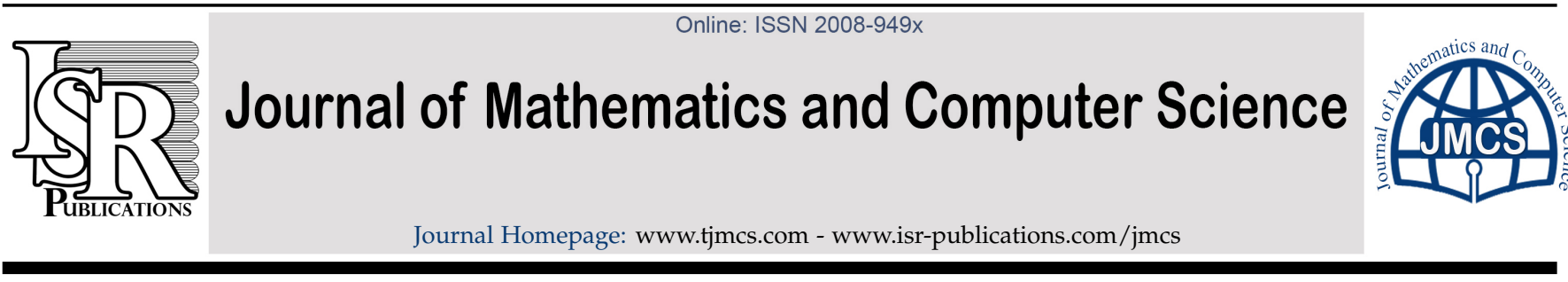

\title{
Common fixed point theorems for two pairs of self-mappings in partial metric space using $C$-class functions on $(\psi, \varphi)$-contractive condition
}

\author{
Zead Mustafaa,b,*, M. M. M. Jaradat ${ }^{\mathrm{a}}$, Arslan Ansaric ${ }^{\mathrm{C}}$, Feng Gu${ }^{\mathrm{d}}$, Hui-hui Zheng ${ }^{\mathrm{d}}$, Stojan Radenoviće ${ }^{\mathrm{e}}$, M. S. \\ Bataineh $^{\dagger}$ \\ ${ }^{a}$ Department of Mathematics, Statistics and Physics, Qatar University, Doha-Qatar. \\ ${ }^{b}$ Department of Mathematics, The Hashemite University, Zarqa 13133, Jordan. \\ ${ }^{c}$ Department of Mathematics, Karaj Branch, Islamic Azad University, Karaj, Iran. \\ ${ }^{d}$ Institute of Applied Mathematics and Department of Mathematics, Hangzhou Normal University, Hangzhou, Zhejiang 310036, China. \\ ${ }^{e}$ Faculty of Mechanical Engineering, University of Belgrade, Serbia. \\ ${ }^{f}$ Department of Mathematics, University of Sharjah, Sharjah, United Arab Emirates.
}

\begin{abstract}
The purpose of this paper is to introduce common fixed point results for two pairs of weakly compatible self-mappings in partial metric space using C-class functions on $(\psi, \varphi)$-contractive condition. Example and application on integral equations are presented to illustrate the main result. Our results extend and generalize well know results in the literature.
\end{abstract}

Keywords: $(\psi, \varphi)$-Contractive mapping, common fixed point, coincidence point, partial metric space, weakly compatible mappings, C-class functions, integral equations.

2010 MSC: 47H10, 54H 25.

(C)2018 All rights reserved.

\section{Introduction and mathematical preliminaries}

In 1994, Matthews [17] introduced the notion of partial metric spaces and obtained various fixed point theorems. In fact, he showed that the Banach contraction mapping theorem can be generalized to the partial metric context.

Later on, Romaguera [30] introduced the notions of 0-Cauchy sequences and 0-complete partial metric spaces and proved some characterizations of partial metric spaces in terms of completeness and 0completeness. Afterwards, several authors obtained some fixed point results for mappings satisfying

\footnotetext{
${ }^{*}$ Corresponding author

Email addresses: zead@qu.edu.qa (Zead Mustafa), mmjst4@qu.edu.qa (M. M. M. Jaradat), analsisamirmath2@gmail.com (Arslan Ansari), gufeng_99@163.com (Feng Gu), zhenghuihui910@sina.com (Hui-hui Zheng), radens@beotel . net (Stojan Radenović), bataineh71@hotmail.com (M. S. Bataineh)
}

doi: 10.22436/jmcs.018.02.09 
different contractive conditions (see [1, 3-9, 13-16, 28-31]). In 2014 the concept of C-class functions (see Definition 1.6) was introduced by Ansari in [3]. For more results on common fixed point for different metric spaces see the references [2, 10-12, 18-27].

The purpose of this paper is to introduce common fixed point results for two pairs of weakly compatible self-mappings in partial metric space using C-class functions on $(\psi, \varphi)$-contractive condition. Example and application on integral equations are presented to illustrate the main result.

Definition 1.1. ([17]). A partial metric on a nonempty set $X$ is a function $p: X \times X \rightarrow R^{+}$such that for all $a, b, c \in X:$

(P1) $p(a, a)=p(a, b)=p(b, b) \Rightarrow a=b$;

(P2) $p(a, a) \leqslant p(a, b)$;

(P3) $p(a, b)=p(b, a)$;

(P4) $p(a, b) \leqslant p(a, c)+p(c, b)-p(c, c)$.

A partial metric space is a pair $(X, p)$ such that $X$ is a nonempty set and $p$ is a partial metric on $X$.

Suppose that $(X, p)$ be a partial metric space, then the function $d_{p}: X \times X \longrightarrow \mathbb{R}^{+}$given by

$$
d_{p}(x, y)=2 p(x, y)-p(x, x)-p(y, y)
$$

is a (usual) metric on $X$. Each partial metric $p$ on $X$ generates a $T_{0}$ topology $\tau_{p}$ on $X$ with a base of the family of open $p$-balls $\left\{B_{p}(x, \varepsilon): x \in X, \varepsilon>0\right\}$, where

$$
B_{p}(x, \varepsilon)=\{y \in X: p(x, y)<p(x, x)+\varepsilon\}
$$

for all $x \in X$ and $\varepsilon>0$.

Definition $1.2([17,29])$. Let $(X, p)$ be a partial metric space. Then

(1) a sequence $\left\{a_{n}\right\}$ in $(X, p)$ converges to a point $a \in X$ if and only if $p(a, a)=\lim _{n \rightarrow \infty} p\left(a_{n}, a\right)$;

(2) a sequence $\left\{a_{n}\right\}$ in $(X, p)$ is called a Cauchy sequence if $\lim _{n, m \rightarrow \infty} p\left(a_{n}, a_{m}\right)$ exists and finite;

(3) $(X, p)$ is said to be complete if every Cauchy sequence $\left\{a_{n}\right\}$ in $X$ converges with respect to $\tau_{p}$ to a point $a \in X$ such that $p(a, a)=\lim _{n, m \rightarrow \infty} p\left(a_{n}, a_{m}\right)$;

(4) a sequence $\left\{a_{n}\right\}$ in $(X, p)$ is called a 0 -Cauchy sequence if $\lim _{n, m \rightarrow \infty} p\left(a_{n}, a_{m}\right)=0$. The space $(X, p)$ is said to be 0 -complete if every 0 -Cauchy sequence in $X$ converges with respect to $\tau_{p}$ to a point $a \in X$ such that $p(a, a)=0$.

Lemma $1.3([17,29])$. Let $(X, p)$ be a partial metric space and $\left\{a_{n}\right\}$ be any sequence in $X$. Then

(i) $\left\{a_{n}\right\}$ is a Cauchy sequence in $(X, p)$ if and only if it is a Cauchy sequence in the metric space $\left(X, d_{p}\right)$;

(ii) the space $(X, p)$ is complete if and only if the metric space $\left(X, \mathrm{~d}_{p}\right)$ is complete;

(iii) every 0 -Cauchy sequence in $(X, p)$ is Cauchy in $\left(X, d_{p}\right)$;

(iv) if $(\mathrm{X}, \mathrm{p})$ is complete, then it is 0-complete.

The converse assertions of (iii) and (iv) do not hold (see example in [30]).

It is easy to see that every closed subset of a 0-complete partial metric space is 0-complete.

Lemma $1.4([1,29])$. Assume $\mathrm{a}_{\mathrm{n}} \rightarrow \mathrm{a}$ as $\mathrm{n} \rightarrow \infty$ in a partial metric space $(\mathrm{X}, \mathrm{p})$ such that $\mathrm{p}(\mathrm{a}, \mathrm{a})=0$. Then $\lim _{n \rightarrow \infty} p\left(a_{n}, b\right)=p(a, b)$ for all $b \in X$.

Lemma $1.5([1,14])$. Suppose that $(X, p)$ be a complete partial metric space. Then

(1) if $p(a, b)=0$, then $a=b$;

(2) if $a \neq b$, then $p(a, b)>0$. 
Definition $1.6([3])$. A mapping $f:[0, \infty) \times[0, \infty) \rightarrow \mathbb{R}$ is called C-class function if it is continuous and satisfies following axioms:

(1) $f(a, b) \leqslant a ;$

(2) $f(a, b)=a$ implies that either $a=0$ or $b=0$; for all $a, b \in[0, \infty)$.

We denote $\mathrm{C}$-class functions as $\mathcal{C}$.

Example 1.7 ([3]). The following functions $f:[0, \infty) \times[0, \infty) \rightarrow \mathbb{R}$ are elements of $\mathcal{C}$ for all $a, b \in[0, \infty)$ :

1. $f(a, b)=a-b, f(a, b)=a \Rightarrow b=0$;

2. $f(a, b)=m a, 0<m<1, f(a, b)=a \Rightarrow a=0$;

3. $f(a, b)=(a+s)^{\left(1 /(1+b)^{r}\right)}-s ; s>1, r \in(0, \infty), f(a, b)=a \Rightarrow b=0$;

4. $f(a, b)=a-\left(\frac{1+a}{2+a}\right)\left(\frac{b}{1+b}\right), f(a, b)=a \Rightarrow b=0$;

5. $f(a, b)=\sqrt[n]{\ln \left(1+a^{\mathfrak{n}}\right)}, f(a, b)=a \Rightarrow a=0$;

6. $f(a, b)=\phi(a), f(a, b)=a \Rightarrow a=0$, here $\phi:[0, \infty) \rightarrow[0, \infty)$ is an upper semicontinuous function such that $\phi(0)=0$, and $\phi(b)<b$ for $b>0$.

Definition 1.8 ([15]). A function $\psi:[0, \infty) \rightarrow[0, \infty)$ is called an altering distance function if it satisfies the following conditions:

1. $\psi$ is continuous and nondecreasing;

2. $\psi(a)=0$ if and only if $a=0$.

We denote by $\Psi$ the set of all altering distance functions.

Definition 1.9. The function $\varphi:[0, \infty) \rightarrow[0, \infty)$ is called an Ultra-altering distance function, if the following conditions hold:

1. $\varphi$ is continuous;

2. $\varphi(b) \neq 0$ when $b \neq 0$.

We denote by $\Phi$ the set of all ultra altering distance functions.

Lemma 1.10 ([28]). Let $(X, p)$ be a partial metric space and $\left\{a_{n}\right\}$ a sequence in $X$ such that $\lim _{n \rightarrow \infty} p\left(a_{n}, a_{n+1}\right)=$ 0 . If $\left\{a_{2 n}\right\}$ is not a Cauchy sequence in $(X, p)$, then there exist $\epsilon>0$ and two sequences $\{m(k)\}$ and $\{n(k)\}$ of positive integers such that $\mathrm{m}(\mathrm{k})>\mathrm{n}(\mathrm{k})>\mathrm{k}$ and the following four sequences tend to $\epsilon>0$, when $\mathrm{k} \rightarrow \infty$.

$$
p\left(a_{2 n(k)}, a_{2 m(k)+1}\right), \quad p\left(a_{2 n(k)}, a_{2 m(k)}\right), \quad p\left(a_{2 n(k)-1}, a_{2 m(k)+1}\right), \quad p\left(a_{2 n(k)-1}, a_{2 m(k)}\right) .
$$

\section{Main results}

The following lemmas will be needed in sequel.

Lemma 2.1. Let $(X, p)$ be a partial metric space and $\left(x_{n}\right)$ be a sequence such that $\lim _{n \rightarrow \infty} p\left(x_{n}, x_{n+1}\right)=0$, then the sequence $\left(\mathrm{x}_{\mathrm{n}}\right)$ is Cauchy sequence iff $\left(\mathrm{x}_{2 \mathrm{n}}\right)$ is Cauchy subsequence.

Proof. Assume that $\left(x_{2 n}\right)$ is Cauchy subsequence. Using the property (P2) of partial metric space and $\lim _{n \rightarrow \infty} p\left(x_{n}, x_{n+1}\right)=0$, we deduce that $\lim _{n \rightarrow \infty} p\left(x_{n}, x_{n}\right)=0$.

Let $m, n \in N$. We will consider three cases:

Case (1). $n$ and $m$ are even. Then there exist $n_{1}$ and $m_{1}$ such that $n=2 n_{1}$ and $m=2 m_{1}$, hence

$$
\lim _{n, m \rightarrow \infty} p\left(x_{n}, x_{m}\right)=\lim _{n_{1}, m_{1} \rightarrow \infty} p\left(x_{2 n_{1}}, x_{2 m_{1}}\right)=0
$$


Case (2). One of $n$ and $m$ is even, say $n$, and the other is odd. Then there exist $n_{1}$ and $m_{1}$ such that $\mathrm{n}=2 \mathrm{n}_{1}$ and $\mathrm{m}=2 \mathrm{~m}_{1}+1$, and so by $(\mathrm{p} 4)$ we get

$$
p\left(x_{n}, x_{m}\right)=p\left(x_{2 n_{1}}, x_{2 m_{1}+1}\right) \leqslant p\left(x_{2 n_{1}}, x_{2 m_{1}}\right)+p\left(x_{2 m_{1}}, x_{2 m_{1}+1}\right)-p\left(x_{2 m_{1}}, x_{2 m_{1}}\right) .
$$

Hence,

$$
\begin{aligned}
\lim _{n \rightarrow m} p\left(x_{n}, x_{m}\right) & =\lim _{n_{1}, m_{1} \rightarrow \infty} p\left(x_{2 n_{1}}, x_{2 m_{1}+1}\right) \\
& \leqslant \lim _{n_{1}, m_{1} \rightarrow \infty} p\left(x_{2 n_{1}}, x_{2 m_{1}}\right)+\lim _{m_{1} \rightarrow \infty} p\left(x_{2 m_{1}}, x_{2 m_{1}+1}\right)-\lim _{m_{1} \rightarrow \infty} p\left(x_{2 m_{1}}, x_{2 m_{1}}\right)=0 .
\end{aligned}
$$

Case (3). $n$ and $m$ are odd. Then there exist $n_{1}$ and $m_{1}$ such that $n=2 n_{1}+1$ and $m=2 m_{1}+1$, so by (p4) we get

$$
\begin{aligned}
p\left(x_{n}, x_{m}\right) & =p\left(x_{2 n_{1}+1}, x_{2 m_{1}+1}\right) \\
& \leqslant p\left(x_{2 n_{1}}, x_{2 n_{1}+1}\right)+p\left(x_{2 n_{1}}, x_{2 m_{1}+1}\right)-p\left(x_{2 n_{1}}, x_{2 n_{1}}\right) \\
& \leqslant p\left(x_{2 n_{1}}, x_{2 n_{1}+1}\right)+p\left(x_{2 n_{1}}, x_{2 m_{1}}\right)+p\left(x_{2 m_{1}}, x_{2 m_{1}+1}\right)-p\left(x_{2 n_{1}}, x_{2 n_{1}}\right)-p\left(x_{2 m_{1}}, x_{2 m_{1}}\right) .
\end{aligned}
$$

Hence,

$$
\begin{aligned}
\lim _{n \rightarrow \infty} p\left(x_{n}, x_{m}\right)= & \lim _{n_{1}, m_{1} \rightarrow \infty} p\left(x_{2 n_{1}+1}, x_{2 m_{1}+1}\right) \\
\leqslant & \lim _{n_{1}, m_{1} \rightarrow \infty} p\left(x_{2 n_{1}}, x_{2 n_{1}+1}\right)+\lim _{m_{1} \rightarrow \infty} p\left(x_{2 n_{1}}, x_{2 m_{1}}\right)+\lim _{m_{1} \rightarrow \infty} p\left(x_{2 m_{1}}, x_{2 m_{1}+1}\right) \\
& -\lim _{n_{1} \rightarrow \infty} p\left(x_{2 n_{1}}, x_{2 n_{1}}\right)-\lim _{m_{1} \rightarrow \infty} p\left(x_{2 m_{1}}, x_{2 m_{1}}\right)=0 .
\end{aligned}
$$

Therefore, the sequence $\left(x_{n}\right)$ is Cauchy sequence.

Lemma 2.2. Let $(X, p)$ be a complete partial metric space and $\left(x_{n}\right)$ a Cauchy sequence with $\lim _{n \rightarrow \infty} p\left(x_{n}, x_{n}\right)=0$, then the sequence $\left(x_{n}\right)$ is 0 -Cauchy sequence. Further if $\left(x_{n}\right)$ converges to $x$, then $\lim _{n \rightarrow \infty} p\left(x_{n}, x\right)=0$

Proof. Since $\left(x_{n}\right)$ is a Cauchy sequence in a complete partial metric space, there exist $x \in X$ such that $\lim _{n, m \rightarrow \infty} p\left(x_{n}, x_{m}\right)=p(x, x)$.

By lemma 1.3, we have $\left(X, d_{\mathfrak{p}}\right)$ is complete metric space and $\left(x_{\mathfrak{n}}\right)$ is Cauchy in $\left(X, d_{\mathfrak{p}}\right)$. Therefore, $\lim _{n, m \rightarrow \infty} d_{p}\left(x_{n}, x_{m}\right)=0$, then $\lim _{n, m \rightarrow \infty}\left[2 p\left(x_{n}, x_{m}\right)-p\left(x_{n}, x_{n}\right)-p\left(x_{m}, x_{m}\right)\right]=0$. But $\lim _{n \rightarrow \infty} p\left(x_{n}, x_{n}\right)=0$, thus $\lim _{n, m \rightarrow \infty} 2 p\left(x_{n}, x_{m}\right)=0$ which implies $0=\lim _{n, m \rightarrow \infty} p\left(x_{n}, x_{m}\right)=p(x, x)$.

Also, $\left(x_{n}\right)$ is Cauchy in a complete metric space $\left(X, d_{p}\right)$, then $\lim _{n \rightarrow \infty} d_{p}\left(x_{n}, x\right)=0$ which implies that $\lim _{n \rightarrow \infty}\left[2 p\left(x_{n}, x\right)-p\left(x_{n}, x_{n}\right)-p(x, x)\right]=0$, thus $\lim _{n \rightarrow \infty} p\left(x_{n}, x\right)=0$.

From Lemma 2.2 and (p2) we deduce the following lemma.

Lemma 2.3. Let $(X, p)$ be a complete partial metric space and $\left(x_{n}\right)$ a Cauchy sequence with $\lim _{n \rightarrow \infty} p\left(x_{n}, x_{n+1}\right)=0$. Then the sequence $\left(x_{n}\right)$ is 0-Cauchy sequence.

Theorem 2.4. Let $\mathrm{F}, \mathrm{G}, \mathrm{S}$, and $\mathrm{T}$ be four self-maps of a complete partial metric space $(\mathrm{X}, \mathrm{p})$ such that

(i) $\mathrm{FX} \subseteq \mathrm{TX}$ and $\mathrm{GX} \subseteq \mathrm{SX}$;

(ii) one of the ranges $S X$ and $T X$ is a closed subset of $(X, p)$;

(iii) the pairs $\{\mathrm{F}, \mathrm{S}\}$ and $\{\mathrm{G}, \mathrm{T}\}$ are weakly compatible and

$$
\psi\left(2 p^{2}(F x, G y)\right) \leqslant f(\psi(\mathcal{M}(x, y)), \varphi(\mathcal{M}(x, y))) \quad \text { for all } x, y \in X
$$

where $\varphi \in \Phi, \psi \in \Psi, f \in \mathcal{C}$ and

$$
\mathcal{M}(x, y)=\max \left\{\begin{array}{c}
p^{2}(S x, T y), p^{2}(F x, S x), p^{2}(G y, T y) \\
p(S x, T y) p(F x, T y), p(\mathcal{F x}, S x) p(F x, T y), \\
p(G y, T y) p(F x, T y), \frac{1}{4}[p(S x, G y)+p(F x, T y)]^{2}
\end{array}\right\}
$$

for all $x, y \in X$. 
Then $\mathrm{F}, \mathrm{G}, \mathrm{S}$, and $\mathrm{T}$ have a unique common fixed point in $\mathrm{X}$.

Proof. Let $x_{0} \in X$ be arbitrary point. Since $F X \subseteq T X$, then there exist a point $x_{1} \in X$ such that $F x_{0}=T x_{1}$, also since $G X \subseteq S X$, then there exist $x_{2} \in X$ such that $G x_{1}=S x_{2}$. Continuing in this process we can construct two sequences $\left\{x_{n}\right\}$ and $\left\{y_{n}\right\}$ in $X$ satisfying

$$
\mathrm{y}_{2 \mathrm{n}}=\mathrm{Fx}_{2 \mathrm{n}}=\mathrm{T} x_{2 \mathrm{n}+1} \quad \text { and } \quad \mathrm{y}_{2 \mathrm{n}+1}=\mathrm{Gx}_{2 \mathrm{n}+1}=\mathrm{S} \mathrm{x}_{2 \mathrm{n}+2}
$$

for all $n \in N$.

The next step, we prove that $\left\{y_{n}\right\}$ is a Cauchy sequence in the partial metric space $(X, p)$. From (2.2) and (2.3) we have

$$
\begin{gathered}
\mathcal{M}\left(x_{2 n}, x_{2 n+1}\right)=\max \left\{\begin{array}{c}
p^{2}\left(S x_{2 n}, T x_{2 n+1}\right), p^{2}\left(F x_{2 n}, S x_{2 n}\right), \\
p^{2}\left(G x_{2 n+1}, T x_{2 n+1}\right), \\
p\left(S x_{2 n}, T x_{2 n+1}\right) p\left(F x_{2 n}, T x_{2 n+1}\right), \\
p\left(F x_{2 n}, S x_{2 n}\right) p\left(F x_{2 n}, T x_{2 n+1}\right), \\
p\left(G x_{2 n+1}, T x_{2 n+1}\right) p\left(F x_{2 n}, T x_{2 n+1}\right), \\
\frac{1}{4}\left[p\left(S x_{2 n}, G x_{2 n+1}\right)+p\left(F x_{2 n}, T x_{2 n+1}\right)\right]^{2}
\end{array}\right\} \\
=\max \left\{\begin{array}{c}
p^{2}\left(y_{2 n-1}, y_{2 n}\right), p^{2}\left(y_{2 n}, y_{2 n-1}\right), p^{2}\left(y_{2 n+1}, y_{2 n}\right), \\
p\left(y_{2 n-1}, y_{2 n}\right) p\left(y_{2 n}, y_{2 n}\right), \\
p\left(y_{2 n}, y_{2 n-1}\right) p\left(y_{2 n}, y_{2 n}\right), \\
p\left(y_{2 n+1}, y_{2 n}\right) p\left(y_{2 n}, y_{2 n}\right), \\
\frac{1}{4}\left[p\left(y_{2 n-1}, y_{2 n+1}\right)+p\left(y_{2 n}, y_{2 n}\right)\right]^{2}
\end{array}\right\} .
\end{gathered}
$$

Note that

$$
p\left(y_{2 n}, y_{2 n}\right) \leqslant p\left(y_{2 n-1}, y_{2 n}\right)
$$

and

$$
p\left(y_{2 n-1}, y_{2 n+1}\right) \leqslant p\left(y_{2 n-1}, y_{2 n}\right)+p\left(y_{2 n}, y_{2 n+1}\right)-p\left(y_{2 n}, y_{2 n}\right)
$$

which implies

$$
p\left(y_{2 n-1}, y_{2 n+1}\right)+p\left(y_{2 n}, y_{2 n}\right) \leqslant p\left(y_{2 n-1}, y_{2 n}\right)+p\left(y_{2 n}, y_{2 n+1}\right)
$$

which yields that,

$$
\frac{p\left(y_{2 n-1}, y_{2 n+1}\right)+p\left(y_{2 n}, y_{2 n}\right)}{2} \leqslant \max \left\{p\left(y_{2 n-1}, y_{2 n}\right), p\left(y_{2 n}, y_{2 n+1}\right)\right\}
$$

and so,

$$
\frac{1}{4}\left[p\left(y_{2 n-1}, y_{2 n+1}\right)+p\left(y_{2 n}, y_{2 n}\right)\right]^{2} \leqslant \max \left\{p^{2}\left(y_{2 n-1}, y_{2 n}\right), p^{2}\left(y_{2 n}, y_{2 n+1}\right)\right\} .
$$

Therefore, using (2.5) and (2.6) the above equation (2.4) becomes

$$
\mathcal{M}\left(x_{2 n}, x_{2 n+1}\right)=\max \left\{p^{2}\left(y_{2 n-1}, y_{2 n}\right), p^{2}\left(y_{2 n}, y_{2 n+1}\right)\right\}
$$

for all $n \in \mathbb{N}$. By using (2.1) with $x=x_{2 n}, y=y_{2 n+1}$, we obtain

$$
\begin{aligned}
\psi\left(2 p^{2}\left(y_{2 n}, y_{2 n+1}\right)\right) & =\psi\left(2 p^{2}\left(F x_{2 n}, G x_{2 n+1}\right)\right) \\
& \leqslant f\left(\psi\left(\mathcal{M}\left(x_{2 n}, x_{2 n+1}\right)\right), \varphi\left(\mathcal{N}\left(x_{2 n}, x_{2 n+1}\right)\right)\right) \\
& \leqslant f\left(\psi\left(\max \left\{p^{2}\left(y_{2 n-1}, y_{2 n}\right), p^{2}\left(y_{2 n}, y_{2 n+1}\right)\right\}\right), \varphi\left(\max \left\{p^{2}\left(y_{2 n-1}, y_{2 n}\right), p^{2}\left(y_{2 n}, y_{2 n+1}\right)\right\}\right)\right) .
\end{aligned}
$$

Analogously we can show that

$$
\begin{aligned}
\psi\left(2 p^{2}\left(y_{2 n+1}, y_{2 n+2}\right)\right) \leqslant & f\left(\psi\left(\max \left\{p^{2}\left(y_{2 n}, y_{2 n+1}\right), p^{2}\left(y_{2 n+1}, y_{2 n+2}\right)\right\}\right)\right. \\
& \left.\varphi\left(\max \left\{p^{2}\left(y_{2 n}, y_{2 n+1}\right), p^{2}\left(y_{2 n+1}, y_{2 n+2}\right)\right\}\right)\right)
\end{aligned}
$$


Note that (2.7) and (2.8) imply that for all $n \geqslant 1$

$$
\psi\left(2 p^{2}\left(y_{n}, y_{n+1}\right)\right) \leqslant f\left(\psi\left(\max \left\{p^{2}\left(y_{n-1}, y_{n}\right), p^{2}\left(y_{n}, y_{n+1}\right)\right\}\right), \varphi\left(\max \left\{p^{2}\left(y_{n-1}, y_{n}\right), p^{2}\left(y_{n}, y_{n+1}\right)\right\}\right)\right)
$$

If there exists $n \in \mathbb{N}$ such that $p^{2}\left(y_{n-1}, y_{n}\right)=0$, then we have $y_{n-1}=y_{n}$. It follows from (2.9), properties of $f$, and the nondecreasing property of $\psi$ that

$$
\psi\left(p^{2}\left(y_{n}, y_{n+1}\right)\right) \leqslant \psi\left(2 p^{2}\left(y_{n}, y_{n+1}\right)\right) \leqslant f\left(\psi\left(p^{2}\left(y_{n}, y_{n+1}\right)\right), \varphi\left(p^{2}\left(y_{n}, y_{n+1}\right)\right)\right) \leqslant \psi\left(p^{2}\left(y_{n}, y_{n+1}\right)\right) .
$$

Hence,

$$
f\left(\psi\left(p^{2}\left(y_{n}, y_{n+1}\right)\right), \varphi\left(p^{2}\left(y_{n}, y_{n+1}\right)\right)\right)=\psi\left(p^{2}\left(y_{n}, y_{n+1}\right)\right) .
$$

So, $\psi\left(p^{2}\left(y_{n}, y_{n+1}\right)\right)=0$ or $\varphi\left(p^{2}\left(y_{n}, y_{n+1}\right)\right)=0$, which implies that $p^{2}\left(y_{n}, y_{n+1}\right)=0$ and so $y_{n}=y_{n+1}$. Thus $y_{n-1}=y_{n}=y_{n+1}$.

Continuing in this process we deduce that $y_{n-1}=y_{n}=y_{n+1}=y_{n+2}=\cdots$. Then $\left\{y_{n}\right\}$ is a Cauchy sequence in $(X, p)$.

Without loss of generality, we may assume that $p^{2}\left(y_{n-1}, y_{n}\right)>0$ for each $n \in \mathbb{N}$. Then from (2.9) and using the fact that $f(s, t) \leqslant s$ for all $s, t \geqslant 0$, we have

$$
\psi\left(2 p^{2}\left(y_{n}, y_{n+1}\right)\right) \leqslant \psi\left(\max \left\{p^{2}\left(y_{n-1}, y_{n}\right), p^{2}\left(y_{n}, y_{n+1}\right)\right\}\right) .
$$

By nondecreasing property of $\psi$ we have

$$
2 p^{2}\left(y_{n}, y_{n+1}\right) \leqslant \max \left\{p^{2}\left(y_{n-1}, y_{n}\right), p^{2}\left(y_{n}, y_{n+1}\right)\right\}
$$

which implies that

$$
\max \left\{p^{2}\left(y_{n-1}, y_{n}\right), p^{2}\left(y_{n}, y_{n+1}\right)\right\}=p^{2}\left(y_{n-1}, y_{n}\right)
$$

Therefore, the sequence $\left\{p^{2}\left(y_{n}, y_{n+1}\right)\right\}$ is bounded below and non-increasing, hence there exist $r \geqslant 0$ such that

$$
\lim _{n \rightarrow \infty} p^{2}\left(y_{n}, y_{n+1}\right)=r .
$$

By taking $n \rightarrow \infty$ in (2.9) and using continuity of $\psi$ and $\varphi$, we deduce that

$$
\psi(r) \leqslant \psi(2 r) \leqslant f(\psi(r), \varphi(r)) \leqslant \psi(r) .
$$

So, $\psi(r)=0$ or $\varphi(r)=0$, which implies that $r=0$. Hence,

$$
\lim _{n \rightarrow \infty} p^{2}\left(y_{n}, y_{n+1}\right)=0, \quad \lim _{n \rightarrow \infty} p^{2}\left(y_{n}, y_{n}\right)=0 \text {. }
$$

Now, we prove that the sequence $\left\{y_{2 n}\right\}$ is a Cauchy in the partial metric space $(X, p)$. Suppose that the sequence $\left\{y_{2 n}\right\}$ is not a Cauchy sequence in $(X, p)$, then there exist $\epsilon>0$ and two sequences $\{m(k)\}$ and $\{n(k)\}$ as in Lemma 1.10 such that all sequences in (1.1) are tend to $\epsilon>0$, when $k \rightarrow \infty$. Now, for $x=x_{2 n(k)}$ and $y=x_{2 m(k)+1}$ in equation (2.1), we get

$$
\psi\left(2 p^{2}\left(F x_{2 n(k)}, G x_{2 m(k)+1}\right)\right) \leqslant f\left(\psi\left(\mathcal{M}\left(x_{2 n(k)}, x_{2 m(k)+1}\right)\right), \varphi\left(\mathcal{M}\left(x_{2 n(k)}, x_{2 m}(k)+1\right)\right)\right),
$$

where

$$
\mathcal{M}\left(x_{2 n(k)}, x_{2 m(k)+1}\right)=\max \left\{\begin{array}{c}
p^{2}\left(S x_{2 n(k)}, T x_{2 m(k)+1}\right), p^{2}\left(F x_{2 n(k)}, S x_{2 n(k)}\right), \\
p^{2}\left(G x_{2 m(k)+1}, T x_{2 m}(k)+1\right), \\
p\left(S x_{2 n(k)}, T x_{2 m(k)+1}\right) p\left(F x_{2 n(k)}, T x_{2 m(k)+1}\right), \\
p\left(F x_{2 n}(k), S x_{2 n(k)}\right) p\left(F x_{2 n}(k), T x_{2 m}(k)+1\right), \\
p\left(G x_{2 m(k)+1}, T x_{2 m(k)+1}\right) p\left(F x_{2 n(k)}, T x_{2 m(k)+1}\right), \\
\frac{1}{4}\left[p\left(S x_{2 n(k)}, G x_{2 m(k)+1}\right)+p\left(F x_{2 n(k)}, T x_{2 m(k)+1)}\right]^{2}\right.
\end{array}\right\}
$$




$$
=\max \left\{\begin{array}{c}
p^{2}\left(y_{2 n(k)-1}, y_{2 m(k)}\right), p^{2}\left(y_{2 n(k)}, y_{2 n(k)-1}\right), \\
p^{2}\left(y_{2 m(k)+1}, y_{2 m(k)}\right), \\
p\left(y_{2 n(k)-1}, y_{2 m(k)}\right) p\left(y_{2 n(k)}, y_{2 m(k)}\right), \\
p\left(y_{2 n(k)}, y_{2 n(k)-1}\right) p\left(y_{2 n(k)}, y_{2 m(k)}\right), \\
p\left(y_{2 m(k)+1}, y_{2 m(k)}\right) p\left(y_{2 n(k)}, y_{2 m(k)}\right), \\
\frac{1}{4}\left[p\left(y_{2 n(k)-1}, y_{2 m(k)+1}\right)+p\left(y_{2 n(k)}, y_{2 m(k)}\right)\right]^{2}
\end{array}\right\} .
$$

Using Lemma 1.10 and equation (2.10) in the above inequality, we have

$$
\lim _{k \rightarrow \infty} \mathcal{M}\left(x_{2 n(k)}, x_{2 m(k)+1}\right) \rightarrow \max \left\{\epsilon^{2}, 0,0, \epsilon^{2}, 0,0, \epsilon^{2}\right\} .
$$

Therefore, taking $k \rightarrow \infty$ in inequality (2.11) and using the properties of $f$ we get

$$
\psi\left(\epsilon^{2}\right) \leqslant \psi\left(2 \epsilon^{2}\right) \leqslant f\left(\psi\left(\epsilon^{2}\right), \varphi\left(\epsilon^{2}\right)\right) \leqslant \psi\left(\epsilon^{2}\right) .
$$

So, $\psi\left(\epsilon^{2}\right)=0$ or $\varphi\left(\epsilon^{2}\right)=0$, hence we get $\epsilon=0$ which contradicts being $\varepsilon>0$. Thus $\left\{y_{2 n}\right\}$ is a Cauchy sequence in $(X, p)$, hence by lemma 2.1 we deduce that the sequence $\left\{y_{n}\right\}$ is Cauchy sequence. Since $(X, p)$ is complete. As a result of Lemma 2.2 we have

$$
p(y, y)=\lim _{n \rightarrow \infty} p\left(y_{n}, y\right)=\lim _{n, m \rightarrow \infty} p\left(y_{n}, y_{m}\right)=0,
$$

and so,

$$
p^{2}(y, y)=\lim _{n \rightarrow \infty} p^{2}\left(y_{n}, y\right)=\lim _{n, m \rightarrow \infty} p^{2}\left(y_{n}, y_{m}\right)=0
$$

This implies that

$$
\lim _{n \rightarrow \infty} p^{2}\left(y_{2 n}, y\right)=\lim _{n \rightarrow \infty} p^{2}\left(y_{2 n+1}, y\right)=0 .
$$

It follows from (2.3) and (2.13) that

$$
\lim _{n \rightarrow \infty} p^{2}\left(F x_{2 n}, y\right)=\lim _{n \rightarrow \infty} p^{2}\left(T x_{2 n+1}, y\right)=0
$$

and

$$
\lim _{n \rightarrow \infty} p^{2}\left(G x_{2 n+1}, y\right)=\lim _{n \rightarrow \infty} p^{2}\left(S x_{2 n+2}, y\right)=0 .
$$

Assume that $S(X)$ is a closed subset of the complete partial metric space $(X, p)$. From (2.14), there exists $u \in X$ such that $y=S u$. We claim that $p^{2}(F u, y)=0$. Otherwise, $p^{2}(F u, y)>0$. By $(p 2)$, (p4), and (2.1) we infer that

$$
\begin{aligned}
p^{2}(y, F u) & \leqslant\left[p\left(y, G x_{2 n+1}\right)+p\left(F u, G x_{2 n+1}\right)-p\left(G x_{2 n+1}, G x_{2 n+1}\right)\right]^{2} \\
& \leqslant p^{2}\left(y, G x_{2 n+1}\right)+p^{2}\left(F u, G x_{2 n+1}\right)+p^{2}\left(G x_{2 n+1}, G x_{2 n+1}\right)+2 p\left(y, G x_{2 n+1}\right) p\left(F u, G x_{2 n+1}\right) \\
& \leqslant p^{2}\left(y, G x_{2 n+1}\right)+p^{2}\left(F u, G x_{2 n+1}\right)+p^{2}\left(y, G x_{2 n+1}\right)+\left[p^{2}\left(y, G x_{2 n+1}\right)+p^{2}\left(F u, G x_{2 n+1}\right)\right] \\
& \left.=3 p^{2}\left(y, G x_{2 n+1}\right)+2 p^{2}\left(F u, G x_{2 n+1}\right)\right),
\end{aligned}
$$

which implies that

$$
\psi\left(p^{2}(y, F u)-3 p^{2}\left(y, y_{2 n+1}\right)\right) \leqslant \psi\left(2 p^{2}\left(\mathcal{F u}, G x_{2 n+1}\right)\right) \leqslant f\left(\psi\left(\mathcal{M}\left(u, x_{2 n+1}\right)\right), \psi\left(\mathcal{M}\left(u, x_{2 n+1}\right)\right)\right) .
$$

On the other hand, it follows from (2.3) and (p4), that

$$
\begin{aligned}
\mathcal{M}\left(u, x_{2 n+1}\right) & =\max \left\{\begin{array}{c}
p^{2}\left(S u, T x_{2 n+1}\right), p^{2}(F u, S u), p^{2}\left(G x_{2 n+1}, T x_{2 n+1}\right), \\
p\left(S u, T x_{2 n+1}\right) p\left(F u, T x_{2 n+1}\right), p(F u, S u) p\left(F u, T x_{2 n+1}\right), \\
p\left(G x_{2 n+1}, T x_{2 n+1}\right) p\left(F u, T x_{2 n+1}\right), \\
\frac{1}{4}\left[p\left(S u, G x_{2 n+1}\right)+p\left(F u, T x_{2 n+1}\right)\right]^{2}
\end{array}\right\} \\
& =\max \left\{\begin{array}{c}
p^{2}\left(y, y_{2 n}\right), p^{2}(F u, y), p^{2}\left(y_{2 n+1}, y_{2 n}\right), \\
p\left(y, y_{2 n}\right) p\left(F u, y_{2 n}\right), p(F u, y) p\left(F u, y_{2 n}\right), \\
p\left(y_{2 n+1}, y_{2 n}\right) p\left(F u, y_{2 n}\right), \\
\frac{1}{4}\left[p\left(y, y_{2 n+1}\right)+p\left(F u, y_{2 n}\right)\right]^{2}
\end{array}\right\} .
\end{aligned}
$$


By taking the limit as $n \rightarrow \infty$ in (2.16), and using (2.12) and (2.13) we deduce that

$$
\lim _{n \rightarrow \infty} \mathcal{M}\left(u, x_{2 n+1}\right)=p^{2}(F u, y) .
$$

By taking the limit as $n \rightarrow \infty$ in (2.15) and using (2.13) and the continuity of $\psi$ we obtain

$$
\psi\left(\lim _{n \rightarrow \infty} p^{2}(y, F u)-3 p^{2}\left(y, y_{2 n+1}\right)\right) \leqslant f\left(\lim _{n \rightarrow \infty} \psi\left(\mathcal{M}\left(u, x_{2 n+1}\right)\right), \lim _{n \rightarrow \infty} \varphi\left(\mathcal{M}\left(u, x_{2 n+1}\right)\right)\right)
$$

which implies, by (2.17), that,

$$
\psi\left(p^{2}(y, F u)\right) \leqslant f\left(\psi\left(p^{2}(F u, y)\right), \varphi\left(p^{2}(F u, y)\right)\right) \leqslant \psi\left(p^{2}(y, F u)\right) .
$$

Hence, $\psi\left(p^{2}(y, F u)\right)=0$ or $\varphi\left(p^{2}(y, F u)\right)=0$, which implies that $p^{2}(y, F u)=0$, so

$$
\mathrm{Fu}=\mathrm{y}=\mathrm{Su} .
$$

That is, $u$ is a coincidence point of $F$ and $S$. In view of $y=F u \in F X \subseteq T X$, we deduce that there exists $v \in X$ such that $y=T v$.

Now we show that $p^{2}(G v, y)=0$. Otherwise $p^{2}(G v, y)>0$. Using (2.1) we infer that

$$
\psi\left(2 p^{2}(y, G v)\right)=\psi\left(2 p^{2}(F u, G v)\right) \leqslant f(\psi(\mathcal{M}(u, v)), \varphi(\mathcal{M}(u, v))) .
$$

In light of $y=S u=F u=T v$, we get that

$$
\begin{aligned}
\mathcal{M}(u, v) & =\max \left\{\begin{array}{c}
p^{2}(\mathrm{Su}, \mathrm{T} v), p^{2}(\mathrm{Fu}, \mathrm{Su}), \mathrm{p}^{2}(\mathrm{G} v, \mathrm{~T} v), \\
p(\mathrm{Su}, \mathrm{T} v) \mathrm{p}(\mathrm{Fu}, \mathrm{T} v), p(\mathcal{F u}, \mathrm{Su}) \mathrm{p}(\mathrm{Fu}, \mathrm{T} v), \\
p(\mathrm{G} v, \mathrm{~T} v) p(\mathrm{Fu}, \mathrm{T} v), \frac{1}{4}[\mathrm{p}(\mathrm{Su}, \mathrm{G} v)+p(\mathrm{Fu}, \mathrm{T} v)]^{2}
\end{array}\right\} \\
& =\max \left\{\begin{array}{c}
p^{2}(y, y), p^{2}(y, y), p^{2}(G v, y), p^{2}(y, y), \\
\left.p^{2}(y, y), p(G v, y) p(y, y), \frac{1}{4}[p(y, G v)+p(y, y)]^{2}\right\}
\end{array}\right\} \\
& =p^{2}(G v, y)(\operatorname{since} p(y, y)=0) .
\end{aligned}
$$

By using (2.19), (2.20), and the property of $f$, we deduce that

$$
\psi\left(p^{2}(G v, y)\right) \leqslant \psi\left(2 p^{2}(G v, y)\right) \leqslant f\left(\psi\left(p^{2}(G v, y)\right), \varphi\left(p^{2}(G v, y)\right)\right) \leqslant \psi\left(p^{2}(G v, y)\right) .
$$

Hence, $\psi\left(p^{2}(G v, y)\right)=0$ or $\varphi\left(p^{2}(G v, y)\right)=0$, which implies that $p^{2}(G v, y)=0$ and so

$$
\mathrm{G} v=\mathrm{y}=\mathrm{T} v .
$$

That is, $v$ is a coincidence point of $G$ and $T$. Since the pair $\{F, S\}$ is weakly compatible, it follows from (2.18) that

$$
\mathrm{Fy}=\mathcal{F S} \mathrm{u}=\mathcal{S F u}=\mathrm{Sy}
$$

Now we show that $p^{2}(F y, y)=0$.

$$
\begin{aligned}
p^{2}(F y, y) & \leqslant\left[p\left(F y, y_{2 n+1}\right)+p\left(y_{2 n+1}, y\right)-p\left(y_{2 n+1}, y_{2 n+1}\right)\right]^{2} \\
& \leqslant p^{2}\left(F y, y_{2 n+1}\right)+p^{2}\left(y_{2 n+1}, y\right)+p^{2}\left(y_{2 n+1}, y_{2 n+1}\right)+2 p\left(F y, y_{2 n+1}\right) p\left(y_{2 n+1}, y\right) \\
& =p^{2}\left(F y, G x_{2 n+1}\right)+p^{2}\left(y_{2 n+1}, y\right)+p^{2}\left(y_{2 n+1}, y_{2 n+1}\right)+2 p\left(F y, G x_{2 n+1}\right) p\left(y_{2 n+1}, y\right) \\
& \leqslant p^{2}\left(F y, G x_{2 n+1}\right)+p^{2}\left(y_{2 n+1}, y\right)+p^{2}\left(y_{2 n+1}, y_{2 n+1}\right)+\left[p^{2}\left(F y, G_{2 n+1}\right)+p^{2}\left(y_{2 n+1}, y\right)\right] \\
& =2 p^{2}\left(F y, G x_{2 n+1}\right)+2 p^{2}\left(y_{2 n+1}, y\right)+p^{2}\left(y_{2 n+1}, y_{2 n+1}\right)
\end{aligned}
$$


which implies that

$$
\begin{aligned}
& \psi\left(p^{2}(F y, y)-2 p^{2}\left(y_{2 n+1}, y\right)-p^{2}\left(y_{2 n+1}, y_{2 n+1}\right)\right) \\
& \quad \leqslant \psi\left(2 p^{2}\left(F y, G x_{2 n+1}\right)\right) \leqslant f\left(\psi\left(\mathcal{M}\left(y, x_{2 n+1}\right)\right), \varphi\left(\mathcal{M}\left(y, x_{2 n+1}\right)\right)\right)
\end{aligned}
$$

On the other hand,

$$
\begin{aligned}
& \mathcal{M}\left(y, x_{2 n+1}\right)=\max \left\{\begin{array}{c}
p^{2}\left(S y, T x_{2 n+1}\right), p^{2}(F y, S y), p^{2}\left(G x_{2 n+1}, T x_{2 n+1}\right), \\
p\left(S y, T x_{2 n+1}\right) p\left(F y, T x_{2 n+1}\right), p(F y, S y) p\left(F y, T x_{2 n+1}\right), \\
p\left(G x_{2 n+1}, T x_{2 n+1}\right) p\left(F y, T x_{2 n+1}\right), \\
\frac{1}{4}\left[p\left(S y, G x_{2 n+1}\right)+p\left(F y, T x_{2 n+1}\right)\right]^{2} \\
=
\end{array}\right\} \\
& \max \left\{\begin{array}{c}
p^{2}\left(F y, y_{2 n}\right), p^{2}(F y, F y), p^{2}\left(y_{2 n+1}, y_{2 n}\right), \\
p^{2}\left(F y, y_{2 n}\right), p(F y, F y) p\left(F y, y_{2 n}\right), \\
p\left(y_{2 n+1}, y_{2 n}\right) p\left(F y, y_{2 n}\right), \\
\frac{1}{4}\left[p\left(F y, y_{2 n+1}\right)+p\left(F y, y_{2 n}\right)\right]^{2}
\end{array}\right\} .
\end{aligned}
$$

Letting $n \rightarrow \infty$ in (2.24), and using (2.10), we get that

$$
\lim _{n \rightarrow \infty} \mathcal{M}\left(y, x_{2 n+1}\right)=p^{2}(F y, y) \text {. }
$$

By taking the limit as $n \rightarrow \infty$ in (2.23), and in view of (2.25), (2.10), (2.13), and the property of $\psi$, we obtain

$$
\psi\left(p^{2}(F y, y)\right) \leqslant \psi\left(2 p^{2}(F y, y)\right) \leqslant f\left(\psi\left(p^{2}(F y, y)\right), \varphi\left(p^{2}(F y, y)\right)\right) \leqslant \psi\left(p^{2}(F y, y)\right) .
$$

So, $\psi\left(p^{2}(F y, y)\right)=0$ or $\varphi\left(p^{2}(F y, y)\right)=0$, which gives that $p^{2}(F y, y)=0$, and so by (2.22),

$$
F y=y=S y .
$$

Since the pair $\{G, T\}$ is weakly compatible, it follows from (2.21) that

$$
\mathrm{Gy}=\mathrm{GT} v=\mathrm{TG} v=\mathrm{Ty} .
$$

We now prove that $p^{2}(y, G y)=0$. By virtue of (2.1) and (2.26), we obtain

$$
\psi\left(2 p^{2}(y, G y)\right)=\psi\left(2 p^{2}(F y, G y)\right) \leqslant f(\psi(\mathcal{M}(y, y)), \varphi(\mathcal{M}(y, y))) .
$$

On the other hand, using (2.2), (2.26), (2.27), (2.12), and (p2), we have that

$$
\begin{aligned}
\mathcal{M}(y, y) & =\max \left\{\begin{array}{c}
p^{2}(S y, T y), p^{2}(F y, S y), p^{2}(G y, T y), \\
p(S y, T y) p(F y, T y), p(\mathcal{F} y, S y) p(F y, T y), \\
p(G y, T y) p(F y, T y), \frac{1}{4}[p(S y, G y)+p(F y, T y)]^{2}
\end{array}\right\} \\
& =\max \left\{\begin{array}{c}
p^{2}(y, G y), p^{2}(y, y), p^{2}(G y, G y), p^{2}(y, G y), \\
p(y, y) p(y, G y), p(G y, G y) p(y, G y), \\
\frac{1}{4}[p(y, G y)+p(y, G y)]^{2}
\end{array}\right\}=p^{2}(y, G y) .
\end{aligned}
$$

Now using (2.28) and the properties of $f$, we deduce that

$$
\begin{aligned}
\psi\left(p^{2}(y, G y)\right) \leqslant \psi\left(2 p^{2}(y, G y)\right) & \leqslant f(\psi(\mathcal{M}(y, y)), \varphi(\mathcal{M}(y, y))) \\
& \leqslant f\left(\psi\left(p^{2}(y, G y)\right), \varphi\left(p^{2}(y, G y)\right)\right) \leqslant \psi\left(p^{2}(y, G y)\right),
\end{aligned}
$$

which implies $f\left(\psi\left(p^{2}(y, G y)\right), \varphi\left(p^{2}(y, G y)\right)\right)=\psi\left(p^{2}(y, G y)\right)$. So, $\psi\left(p^{2}(y, G y)\right)=0$ or $\varphi\left(p^{2}(y, G y)\right)=0$, hence $p^{2}(y, G y)=0$. Therefore

$$
\mathrm{Gy}=\mathrm{y}=\mathrm{T} y \text {. }
$$


Now, combining (2.26) and (2.29), we obtain

$$
\mathrm{y}=\mathrm{Fy}=\mathrm{Gy}=\mathrm{Sy}=\mathrm{Ty} .
$$

That is, $y$ is a common fixed point of $F, G, S$, and $T$. To prove the uniqueness, suppose that $z$ is another common fixed points of $F, G, S$ and $T$, and $z \neq y$, then using the contractive condition (2.1), (2.2), and (p2), we have

$$
\begin{aligned}
\psi\left(2 p^{2}(y, z)\right) \leqslant \psi\left(p^{2}(y, z)\right)= & \psi\left(2 p^{2}(F y, G z)\right) \quad \\
\leqslant & f\left(\psi\left(\max \left\{\begin{array}{r}
p^{2}(S y, T z), p^{2}(F y, S y), p^{2}(G z, T z), \\
p(S y, T z) p(F y, T z), p(\mathcal{F} y, S y) p(F y, T z), \\
p(G z, T z) p(F y, T z), \frac{1}{4}[p(S y, G z)+p(F y, T z)]^{2}
\end{array}\right\}\right),\right. \\
& \left.\varphi\left(\max \left\{\begin{array}{c}
p^{2}(S y, T z), p^{2}(F y, S y), p^{2}(G z, T z), \\
p(S y, T z) p(F y, T z), p(\mathcal{F} y, S y) p(F y, T z), \\
p(G z, T z) p(F y, T z), \frac{1}{4}[p(S y, G z)+p(F y, T z)]^{2}
\end{array}\right\}\right)\right) \\
= & f\left(\psi\left(\max \left\{\begin{array}{c}
p^{2}(y, z), p^{2}(y, y), p^{2}(z, z), p^{2}(y, z), \\
p(y, y) p(y, z), p(z, z) p(y, z), p^{2}(y, z)
\end{array}\right\}\right),\right. \\
& \left.\varphi\left(\max \left\{\begin{array}{c}
p^{2}(y, z), p^{2}(y, y), p^{2}(z, z), p^{2}(y, z), \\
p(y, y) p(y, z), p(z, z) p(y, z), p^{2}(y, z)
\end{array}\right\}\right)\right) \\
\leqslant & f\left(\psi\left(\max \left\{\begin{array}{c}
p^{2}(y, z), p^{2}(y, z), p^{2}(y, z), p^{2}(y, z), \\
p(y, z) p(y, z), p(y, z) p(y, z), p^{2}(y, z)
\end{array}\right\}\right),\right. \\
& \left.\psi\left(\max \left\{\begin{array}{c}
p^{2}(y, z), p^{2}(y, z), p^{2}(y, z), p^{2}(y, z), \\
p(y, z) p(y, z), p(y, z) p(y, z), p^{2}(y, z)
\end{array}\right\}\right)\right) \\
= & f\left(\psi\left(p^{2}(y, z)\right), \varphi\left(p^{2}(y, z)\right) \leqslant \psi\left(p^{2}(y, z)\right) .\right.
\end{aligned}
$$

So, $\psi\left(p^{2}(y, z)\right)=0$ or $\varphi\left(p^{2}(y, z)\right)=0$, hence $p^{2}(y, z)=0$, which is a contradiction. Thus it should be $z=y$. Consequently, $F, G, S$, and $T$ have a unique common fixed point. Now, if $T(X)$ is a closed subset of the complete partial metric space $(X, p)$, then the proof is similar to the above arguments. This completes the proof.

\section{Some corollaries and examples}

In Theorem 2.4, by taking $\psi(t)=I$ and $f(a, b)=\phi(a)$, where $\phi:[0, \infty) \rightarrow[0, \infty)$ is a continuous function such that $\phi(a)=a$, iff $a=0, \phi(a)<a$ for $a>0$, and $\sum_{n \geqslant 1}\left[\phi^{n}(a)\right]^{\frac{1}{2}}$ converges for all $a \geqslant 0$ we get the following corollary which is a generalization of Theorem 2.1 in [31].

Corollary 3.1. Let $\mathrm{F}, \mathrm{G}, \mathrm{S}$, and $\mathrm{T}$ be four self-maps of a complete partial metric space $(\mathrm{X}, \mathrm{p})$ such that

(i) $\mathrm{FX} \subseteq \mathrm{TX}$ and $\mathrm{GX} \subseteq \mathrm{SX}$;

(ii) one of the ranges $S X$ and $T X$ is a closed subset of $(X, p)$;

(iii) the pairs $\{\mathrm{F}, \mathrm{S}\}$ and $\{\mathrm{G}, \mathrm{T}\}$ are weakly compatible and

$$
\left.2 p^{2}(F x, G y)\right) \leqslant \phi(\mathcal{M}(x, y)), \quad \forall x, y \in X
$$

where $\phi$ is as above and $\mathcal{M}(x, y)$ is as in (2.2) of Theorem 2.4 for all $x, y \in X$.

Then $\mathrm{F}, \mathrm{G}, \mathrm{S}$, and $\mathrm{T}$ have a unique common fixed point in $\mathrm{X}$.

Corollary 3.2. Let $\mathrm{F}, \mathrm{G}, \mathrm{S}$, and $\mathrm{T}$ be four self-maps of a complete partial metric space $(\mathrm{X}, \mathrm{p})$ such that

1. $\mathrm{FX} \subseteq \mathrm{TX}$ and $\mathrm{GX} \subseteq \mathrm{SX}$;

2. one of the ranges $S X$ and $T X$ is a closed subset of $(X, p)$; 
3. the pairs $\{\mathrm{F}, \mathrm{S}\}$ and $\{\mathrm{G}, \mathrm{T}\}$ are weakly compatible and

$$
\begin{aligned}
2 p^{2}(F x, G y) \leqslant & a_{1} p^{2}(S x, T y)+a_{2} p^{2}(F x, S x)+a_{3} p^{2}(G y, T y) \\
& +a_{4} p(S x, T y) p(F x, T y)+a_{5} p(F x, S x) p(F x, T y)+a_{6} p(G y, T y) p(F x, T y)
\end{aligned}
$$

holds for all $x, y \in X$, where $a_{i} \geqslant 0(i=1,2,3, \cdots, 6)$ with $a_{1}+a_{2}+a_{3}+a_{4}+a_{5}+a_{6}<1$.

Then $\mathrm{F}, \mathcal{G}, \mathrm{S}$, and $\mathrm{T}$ have a unique common fixed point in $\mathrm{X}$.

Proof. Let

$$
\mathcal{M}(x, y)=\max \left\{\begin{array}{c}
p^{2}(S x, T y), p^{2}(F x, S x), p^{2}(G y, T y), \\
p(S x, T y) p(F x, T y), p(\mathcal{F} x, S x) p(F x, T y), \\
p(G y, T y) p(F x, T y), \frac{1}{4}[p(S x, G y)+p(F x, T y)]^{2}
\end{array}\right\}
$$

for all $x, y \in X$. Then we have

$$
\begin{aligned}
& a_{1} p^{2}(S x, T y)+a_{2} p^{2}(F x, S x)+a_{3} p^{2}(G y, T y)+a_{4} p(S x, T y) p(F x, T y) \\
& \quad+a_{5} p(F x, S x) p(F x, T y)+a_{6} p(G y, T y) p(F x, T y) \leqslant\left(a_{1}+a_{2}+a_{3}+a_{4}+a_{5}+a_{6}\right) \mathcal{M}(x, y) .
\end{aligned}
$$

So, if the condition (3.1) hold, then

$$
2 p^{2}(F x, G y) \leqslant\left(a_{1}+a_{2}+a_{3}+a_{4}+a_{5}+a_{6}\right) \mathcal{M}(x, y) .
$$

Let $k=a_{1}+a_{2}+a_{3}+a_{4}+a_{5}+a_{6}$ and $\phi(s)=k s$, then the result is obtained from Corollary 3.1.

Remark 3.3. In Theorem 2.4 if we take

1. $S=T$, or

2. $S=T$ and $F=G$, or

3. $S=T=I$, where $I$ is the identity mapping, or

4. $\mathrm{S}=\mathrm{T}=\mathrm{I}$, and $\mathrm{F}=\mathrm{G}$, where $\mathrm{I}$ is the identity mapping,

then we get several new results of unique common fixed point for two and three mappings, and a unique fixed point for one mapping.

By taking $f(a, b)=a-\left(\frac{1+a}{2+a}\right)\left(\frac{b}{1+b}\right)$ in Theorem 2.4 we deduce the following corollary.

Corollary 3.4. Let $\mathrm{F}, \mathrm{G}, \mathrm{S}$, and $\mathrm{T}$ be four self-maps of a complete partial metric space $(\mathrm{X}, \mathrm{p})$ such that

(i) $\mathrm{FX} \subseteq \mathrm{TX}$ and $\mathrm{GX} \subseteq \mathrm{SX}$;

(ii) one of the ranges $S X$ and $T X$ is a closed subset of $(X, p)$;

(iii) the pairs $\{\mathrm{F}, \mathrm{S}\}$ and $\{\mathrm{G}, \mathrm{T}\}$ are weakly compatible and

$$
\psi\left(2 p^{2}(F x, G y)\right) \leqslant \psi(\mathcal{M}(x, y))-\left(\frac{1+\psi(\mathcal{M}(x, y))}{2+\psi(\mathcal{N}(x, y))}\right)\left(\frac{\varphi(\mathcal{M}(x, y)}{1+\varphi(\mathcal{M}(x, y)}\right)
$$

where $\varphi \in \Phi, \psi \in \Psi, f \in \mathcal{C}$, and $\mathcal{M}(x, y)$ is as in (2.2) of Theorem 2.4 for all $x, y \in X$.

Then $\mathrm{F}, \mathrm{G}, \mathrm{S}$, and $\mathrm{T}$ have a unique common fixed point in $\mathrm{X}$.

By taking $F(a, b)=\sqrt[n]{\ln \left(1+a^{n}\right)}$ in Theorem 2.4 we deduce the following corollary.

Corollary 3.5. Let $\mathrm{F}, \mathrm{G}, \mathrm{S}$, and $\mathrm{T}$ be four self-maps of a complete partial metric space $(\mathrm{X}, \mathrm{p})$ such that

(i) $\mathrm{FX} \subseteq \mathrm{TX}$ and $\mathrm{GX} \subseteq \mathrm{SX}$;

(ii) one of the ranges $S X$ and $T X$ is a closed subset of $(X, p)$; 
(iii) the pairs $\{\mathrm{F}, \mathrm{S}\}$ and $\{\mathrm{G}, \mathrm{T}\}$ are weakly compatible and

$$
\psi\left(2 p^{2}(F x, G y)\right) \leqslant \sqrt[n]{\ln \left(1+(\psi(\mathcal{M}(x, y)))^{n}\right)},
$$

where, $\psi \in \Psi, f \in \mathcal{C}$, and $\mathcal{M}(x, y)$ is as in (2.2) of Theorem 2.4 for all $x, y \in X$.

Then $\mathrm{F}, \mathrm{G}, \mathrm{S}$, and $\mathrm{T}$ have a unique common fixed point in $\mathrm{X}$.

By taking $f(a, b)=(a+3)^{\left(1 /(1+b)^{2}\right)}-3$ in Theorem 2.4 we deduce the following corollary.

Corollary 3.6. Let $\mathrm{F}, \mathrm{G}, \mathrm{S}$, and $\mathrm{T}$ be four self-maps of a complete partial metric space $(\mathrm{X}, \mathrm{p})$ such that

(i) $\mathrm{FX} \subseteq \mathrm{TX}$ and $\mathrm{GX} \subseteq \mathrm{SX}$;

(ii) one of the ranges $S X$ and $T X$ is a closed subset of $(X, p)$;

(iii) the pairs $\{\mathrm{F}, \mathrm{S}\}$ and $\{\mathrm{G}, \mathrm{T}\}$ are weakly compatible and

$$
\psi\left(2 p^{2}(F x, G y)\right) \leqslant(\psi(\mathcal{M}(x, y))+3)^{\left(1 /(1+\varphi(\mathcal{M}(x, y)))^{2}\right)}-3
$$

where $\varphi \in \Phi, \psi \in \Psi, f \in \mathcal{C}$, and $\mathcal{M}(x, y)$ is as in (2.2) of Theorem 2.4 for all $x, y \in X$.

Then $\mathrm{F}, \mathrm{G}, \mathrm{S}$, and $\mathrm{T}$ have a unique common fixed point in $\mathrm{X}$.

Remark 3.7. In Corollaries 3.4, 3.5, and 3.6 if we take

1. $\mathrm{S}=\mathrm{T}$, or

2. $S=T$ and $F=G$, or

3. $\mathrm{S}=\mathrm{T}=\mathrm{I}$, where $\mathrm{I}$ is the identity mapping, or

4. $S=T=I$, and $F=G$, where $I$ is the identity mapping,

then we get several new results of unique common fixed point for two and three mappings and a unique fixed point for one mapping.

In Theorem 2.4 , by taking $\psi(t)=t$ we get the following corollary.

Corollary 3.8. Let $\mathrm{F}, \mathrm{G}, \mathrm{S}$, and $\mathrm{T}$ be four self-maps of a complete partial metric space $(\mathrm{X}, \mathrm{p})$ such that

(i) $\mathrm{FX} \subseteq \mathrm{TX}$ and $\mathrm{GX} \subseteq \mathrm{SX}$;

(ii) one of the ranges $S X$ and $T X$ is a closed subset of $(X, p)$;

(iii) the pairs $\{\mathrm{F}, \mathrm{S}\}$ and $\{\mathrm{G}, \mathrm{T}\}$ are weakly compatible and

$$
2 p^{2}(F x, G y) \leqslant f(\mathcal{M}(x, y), \varphi(\mathcal{M}(x, y))), \quad \forall x, y \in X,
$$

where $\varphi \in \Phi$ and $\mathrm{f} \in \mathcal{C}$ and $\mathcal{M}(\mathrm{x}, \mathrm{y})$ is as in (2.2) of Theorem 2.4 for all $\mathrm{x}, \mathrm{y} \in \mathrm{X}$.

Then $\mathrm{F}, \mathrm{G}, \mathrm{S}$, and $\mathrm{T}$ have a unique common fixed point in $\mathrm{X}$.

Now, we give example to support Theorem 2.4.

Example 3.9. Let $X=[0,1]$, and $(X, d)$ be a partial metric space defined by $p(x, y)=\max \{x, y\}$ for all $x, y \in X$. Let $F, G, S$, and $T$ be four self mappings defined by

$$
\mathrm{F} x=\frac{x^{2}}{4}, \quad \mathrm{G} x=\frac{x}{2}, \quad \mathrm{~S} x=x^{2}, \quad \mathrm{~T} x=\frac{3 x}{4}, \quad \text { for all } x \in[0,1] .
$$

Clearly, the subspace $S X=X$ is closed, $F X \subset S X$ and $G X \subset T X$. Also, it is easy to show that the pairs $\{F, S\}$ and $\{G, T\}$ are weakly compatible. In order to check condition (2.1) for all $x, y \in X$ and $\psi(t)=t^{3}$, $\varphi(t)=\sqrt{t}$ for all $t \in \mathbb{R}^{+}$and $f(a, b)=\frac{8 a}{10}$ we consider the following two cases: 
Case 1. If $y \leqslant \frac{x^{2}}{2}$, then

$$
p^{2}(F x, G y)=\left(p\left(\frac{x^{2}}{4}, \frac{y}{2}\right)\right)^{2}=\left(\max \left\{\frac{x^{2}}{4}, \frac{y}{2}\right\}\right)^{2}=\frac{x^{4}}{16}
$$

and

$$
\left.p^{2}(F x, S x)=\left(p\left(\frac{x^{2}}{4}, x^{2}\right)\right)^{2}=\left(\max \left\{\frac{x^{2}}{4}, x^{2}\right)\right\}\right)^{2}=x^{4}
$$

Hence we deduce that

$$
\begin{aligned}
2 p^{2}(F x, G y) & =\frac{x^{4}}{8}=\frac{1}{8} p^{2}(F x, S x) \\
\psi\left(2 p^{2}(F x, G y)\right) & =\frac{x^{12}}{8^{3}} \leqslant \frac{8}{10} x^{12}=f\left(\psi\left(p^{2}(F x, S x)\right), \varphi\left(p^{2}(F x, S x)\right)\right) \leqslant f(\psi(\mathcal{M}(x, y)), \varphi(\mathcal{M}(x, y))) .
\end{aligned}
$$

Case 2. If $\frac{x^{2}}{2}<y$, then

$$
p^{2}(F x, G y)=p^{2}\left(\frac{x^{2}}{4}, \frac{y}{2}\right)=\left(\max \left\{\frac{x^{2}}{4}, \frac{y}{2}\right\}\right)^{2}=\frac{y^{2}}{4}
$$

and

$$
p^{2}(G y, T y)=p^{2}\left(\frac{y}{2}, \frac{3 y}{4}\right)=\left(\max \left\{\frac{y}{2}, \frac{3 y}{4}\right\}\right)^{2}=\frac{9 y^{2}}{16}
$$

Therefore, we get that

$$
\begin{aligned}
2 p^{2}(F x, G y) & =\frac{y^{2}}{2}=\frac{8}{9} p^{2}(G y, T y) \\
\psi\left(2 p^{2}(F x, G y)\right) & =\frac{y^{6}}{8} \leqslant \frac{8}{10} \frac{9^{3}}{16^{3}} y^{6}=f\left(\psi\left(p^{2}(G y, T y)\right), \varphi\left(p^{2}(G y, T y)\right)\right) \leqslant f(\psi(\mathcal{M}(x, y)), \varphi(\mathcal{M}(x, y))) .
\end{aligned}
$$

Hence, all conditions of Theorem 2.4 are satisfied. Moreover, 0 is the unique common fixed point of F, G, S, and $\mathrm{T}$.

\section{Application to integral equation}

In this section, we will use Theorem 2.4 with $S=T=I$, where I is the identity mapping to show that there is a solution to the following integral equations:

$$
\begin{aligned}
& x(t)=h_{1}(x(t))+\int_{0}^{t} m(t, s) H_{1}(s, x(s)) d s, t \in[0,1], \\
& x(t)=h_{2}(x(t))+\int_{0}^{t} m(t, s) H_{2}(s, x(s)) d s, t \in[0,1],
\end{aligned}
$$

where,

1. $h_{1}(t), h_{2}(t):[0,1] \rightarrow R$ are continuous;

2. $\mathfrak{m}(t, s):[0,1] \times[0,1] \rightarrow \mathrm{R}^{+}$are continuous functions;

3. $H_{i}(t, s):[0,1] \times R \rightarrow R$ are continuous functions for $i=1,2$.

Let $X=C([0,1])$ be the set of all real continuous functions on $[0,1]$, endowed with the partial metric

$$
p(u, v)=\max \left\{\sup _{t \in[0,1]}|u(t)|, \sup _{t \in[0,1]}|v(t)|\right\}, \text { for all } u, v \in X .
$$

Clearly, $(X, p)$ is a complete partial metric space. 
Theorem 4.1. The integral equations (4.1) and (4.2) have a solution $u$ such that $u \in C([0,1])$ if the following conditions are holds:

1. $\sup _{\mathrm{t} \in[0,1]} \mathrm{m}(\mathrm{t}, \mathrm{s}) \leqslant \frac{1}{\sqrt{3}}$

2. $\left|H_{i}(s, t)\right| \leqslant \frac{1}{2}|t| ; i=1,2$;

3. $\left|h_{i}(t)\right| \leqslant \frac{1}{2 \sqrt{3}}|t| ; i=1,2$.

Proof. Define mappings $\mathrm{F}, \mathrm{G}: \mathrm{X} \rightarrow \mathrm{X}$ by

$$
\begin{array}{ll}
F x(t)=h_{1}(x(t))+\int_{0}^{t} m(t, s) H_{1}(s, x(s)) d s, & t \in[0,1], \\
G x(t)=h_{2}(x(t))+\int_{0}^{t} m(t, s) H_{2}(s, x(s)) d s, & t \in[0,1],
\end{array}
$$

and $S=T=I$, where $\mathrm{I}$ is the identity mapping. Clearly the conditions (i) and (ii) of Theorem 2.4 are satisfied also the pairs $(F, S)$ and $(G, T)$ are weakly compatible. Now we prove condition (2.1) of Theorem 2.4 is satisfied. Let $x(t), y(t) \in X$. Then, for all $t \in[0,1]$, we have

$$
\begin{aligned}
|F(x(t))| & \leqslant\left|h_{1}(x(t))\right|+\left|\int_{0}^{t} m(t, s) H_{1}(s, x(s)) d s\right| \\
& \leqslant\left|h_{1}(x(t))\right|+\int_{0}^{t}|m(t, s)|\left|H_{1}(s, x(s))\right| d s \\
& \leqslant\left|h_{1}(x(t))\right|+\int_{0}^{t} \frac{1}{\sqrt{3}}\left|H_{1}(s, x(s))\right| d s \\
& \leqslant \frac{1}{2 \sqrt{3}} \sup _{t \in[0,1]}|x(t)|+\int_{0}^{t} \frac{1}{2 \sqrt{3}} \sup _{t \in[0,1]}|x(t)| \\
& =\frac{1}{2 \sqrt{3}} \sup _{t \in[0,1]} \mid S x\left(t\left|+\int_{0}^{t} \frac{1}{2 \sqrt{3}} \sup _{t \in[0,1]}\right| S x(t) \mid\right. \\
& \leqslant \frac{1}{2 \sqrt{3}} \sup _{t \in[0,1]} \mid S x\left(t\left|+\int_{0}^{1} \frac{1}{2 \sqrt{3}} \sup _{t \in[0,1]}\right| S x(t) \mid\right. \\
& =\frac{1}{\sqrt{3}} \sup _{t \in[0,1]}|S x(t)| .
\end{aligned}
$$

Thus,

Similarly,

$$
\sup _{t \in[0,1]}|F(x(t))| \leqslant \frac{1}{\sqrt{3}} \sup _{t \in[0,1]}|S x(t)| .
$$

Hence,

$$
\sup _{t \in[0,1]}|G(y(t))| \leqslant \frac{1}{\sqrt{3}} \sup _{t \in[0,1]}|\operatorname{Ty}(t)|
$$

which implies,

$$
p(F(x(t)), G(y(t))) \leqslant \frac{1}{\sqrt{3}} p(S x(t), T y(t))
$$

$$
\begin{aligned}
2 p^{2}(F(x(t)), G(y(t))) & \leqslant \frac{2}{3} p^{2}(S x(t), T y(t)) \\
& \leqslant \frac{2}{3} \mathcal{M}(x, y)=\psi(\mathcal{M}(x, y))-\varphi(\mathcal{M}(x, y))=f(\psi(\mathcal{M}(x, y)), \varphi(\mathcal{M}(x, y))) .
\end{aligned}
$$

Therefore, $\psi\left(2 p^{2}(F(x(t)), G(y(t)))\right) \leqslant f(\psi(\mathcal{M}(x, y)), \varphi(\mathcal{N}(x, y)))$, where $f(a, b)=a-b, \psi(t)=t$ and $\varphi(t)=\frac{t}{3}$. Hence, all conditions of Theorem 2.4 hold and the mappings $F, G, S$, and $T$ has a common fixed point $u(t) \in C([0,1])$, which is a solution to the equations (4.1) and (4.2). 


\section{References}

[1] T. Abdeljawad, E. Karapınar, K. Taş, Existence and uniqueness of a common fixed point on partial metric spaces, Appl. Math. Lett., 24 (2011), 1900-1904. 1, 1.4, 1.5

[2] Abdullah, M. Sarwar, Z. Mustafa, M. M. M. Jaradat, Common fixed points of $(\phi, \psi)$-contraction on G-metric space using E.A property, J. Math. Anal., 8 (2017), 136-146. 1

[3] A. H. Ansari, Note on " $\varphi-\psi$-contractive type mappings and related fixed point, 2nd Regional Conf. Math. Appl., PNU, Iran, (2014), 377-380. 1, 1.6, 1.7

[4] H. Aydi, Fixed point theorems for generalized weakly contractive condition in ordered partial metric spaces, J. Nonlinear Anal. Optim., 2 (2011), 269-284.

[5] H. Aydi, Some fixed point results in ordered partial metric spaces, J. Nonlinear Sci. Appl., 4 (2011), $210-217$.

[6] A. Bejenaru, A. Pitea, Fixed point and best proximity point theorems on partial metric spaces, J. Math. Anal., 7 (2016), 25-44.

[7] L. Ćirić, B. Samet, H. Aydi, C. Vetro, Common fixed points of generalized contractions on partial metric spaces and an application, Appl. Math. Comput., 218 (2011), 2398-2406.

[8] A. Erduran, Z. Kadelburg, H. K. Nashine, C. Vetro, A fixed point theorem for ( $\phi, \mathrm{L})$-weak contraction mappings on a partial metric space, J. Nonlinear Sci. Appl., 7 (2014), 196-204.

[9] D. Ilić, V. Pavlović, V. Rakočević, Some new extensions of Banach's contraction principle to partial metric space, Appl. Math. Lett., 24 (2011), 1326-1330. 1

[10] M. M. M. Jaradat, Z. Mustafa, A. H. Ansari, S. Chandok, Ć. Dolićanin, Some approximate fixed point results and application on graph theory for partial ( $\mathrm{h}, \mathrm{F})$-generalized convex contraction mappings with special class of functions on complete metric space, J. Nonlinear Sci. Appl., 10 (2017), 1695-1708. 1

[11] M. M. M. Jaradat, Z. Mustafa, A. H. Ansari, P. S. Kumari, D. Dolicanin-Djekic, H. M. Jaradat, Some fixed point

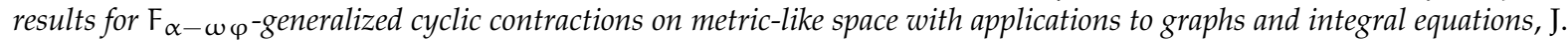
Math. Anal., 8 (2017), 28-45.

[12] M. M. M. Jaradat, Z. Mustafa, S. U. Khan, M. Arshad, J. Ahmad, Some fixed point results on G-metric and $\mathrm{G}_{\mathrm{b}}-$-metric spaces, Demonstr. Math., 50 (2017), 190-207. 1

[13] A. Kaewcharoen, T. Yuying, Unique common fixed point theorems on partial metric spaces, J. Nonlinear Sci. Appl., 7 (2014), 90-101. 1

[14] E. Karapınar, Ćirić types nonunique fixed point theorems on partial metric spaces, J. Nonlinear Sci. Appl., 5 (2012), 74-83. 1.5

[15] M. S. Khan, M. Swalesh, S. Sessa, Fixed point theorems by altering distances between the points, Bull. Austral. Math. Soc., 30 (1984), 1-9. 1.8

[16] V. La Rosa, P. Vetro, Fixed points for Geraghty-contractions in partial metric spaces, J. Nonlinear Sci. Appl., 7 (2014), 1-10. 1

[17] S. G. Matthews, Partial metric topology, Papers on general topology and applications, Flushing, NY, (1992), Ann. New York Acad. Sci., New York Acad. Sci., New York, 728 (1994), 183-197. 1, 1.1, 1.2, 1.3

[18] Z. Mustafa, Common fixed points of weakly compatible mappings in G-metric spaces, Appl. Math. Sci. (Ruse), 6 (2012), 4589-4600. 1

[19] Z. Mustafa, T. V. An, N. V. Dung, Two fixed point theorems for maps on incomplete G-metric spaces, Appl. Math. Sci. (Ruse), 7 (2013), 2271-2281.

[20] Z. Mustafa, M. Arshad, S. U. Khan, J. Ahmad, M. M. M. Jaradat, Common fixed points for multivalued mappings in G-metric spaces with applications, J. Nonlinear Sci. Appl., 10 (2017), 2550-2564.

[21] Z. Mustafa, H. Aydi, E. Karapınar, On common fixed points in G-metric spaces using (E.A) property, Comput. Math. Appl., 64 (2012), 1944-1956.

[22] Z. Mustafa, M. M. M. Jaradat, A. H. Ansari, B. Z. Popović, H. Jaradat, C-class functions with new approach on coincidence point results for generalized $(\psi, \varphi)$-weakly contractions in ordered b-metric spaces, SpringerPlus, 5 (2016), $1-18$.

[23] Z. Mustafa, M. M. M. Jaradat, H. M. Jaradat, Some common fixed point results of graphs on b-metric space, J. Nonlinear Sci. Appl., 9 (2016), 4838-4851.

[24] Z. Mustafa, M. M. M. Jaradat, H. M. Jaradat, A remarks on the paper "Some fixed point theorems for generalized contractive mappings in complete metric spaces", J. Math. Anal., 8 (2017), 17-22.

[25] Z. Mustafa, M. M. M. Jaradat, E. Karapınar, A new fixed point result via property $\mathrm{P}$ with an application, J. Nonlinear Sci. Appl., 10 (2017), 2066-2078.

[26] Z. Mustafa, J. R. Roshan, V. Parvaneh, Z. Kadelburg, Some common fixed point results in ordered partial b-metric spaces, J. Inequal. Appl., 2013 (2013), 26 pages.

[27] Z. Mustafa, J. R. Roshan, V. Parvaneh, Z. Kadelburg, Fixed point theorems for weakly T-Chatterjea and weakly T-Kannan contractions in b-metric spaces, J. Inequal. Appl., 2014 (2014), 14 pages. 1

[28] H. K. Nashine, Z. Kadelburg, S. Radenović, Common fixed point theorems for weakly isotone increasing mappings in ordered partial metric spaces, Math. Comput. Modelling, 57 (2013), 2355-2365. 1, 1.10

[29] S. Oltra, O. Valero, Banach's fixed point theorem for partial metric spaces, Rend. Istit. Mat. Univ. Trieste, 36 (2004), 17-26. 1.2, 1.3, 1.4 
[30] S. Romaguera, A Kirk type characterization of completeness for partial metric spaces, Fixed Point Theory Appl., 2010 (2010), 6 pages. 1,1

[31] H.-H. Zheng, F. Gu, Some results of common fixed point for four self-maps satisfying a new $\Psi$-contractive condition in partial metric spaces, J. Nonlinear Sci. Appl., 9 (2016), 2258-2272. 1, 3 